\title{
PROCESSO DE SOLDAGEM POR FRICÇÃO COM PINO CONSUMÍVEL SFPC APLICADO NO AÇO INOXIDÁVEL DUPLEX SAF 2205
}

\author{
Douglas Martinazzi ' \\ Cleber Rodrigo de Lima Lessa ${ }^{2}$ \\ Arlan Pacheco Figueiredo ${ }^{2}$ \\ Afonso Reguly '
}

\section{Resumo}

A presente investigação demonstra a utilização do processo de Soldagem por Fricção com Pino Consumível (SFPC), ou Friction Hydro Pillar Processing (FHPP), para reparar estruturas que contenham o aço inoxidável duplex SAF 2205 (AID UNS S3 I 803) como material base. Para tanto, foi realizado um estudo preliminar relativo ao mesmo material reparado com o processo Tungsten Inert Gas (TIG). Após a obtenção das juntas reparadas em ambos os processos, estas passaram por rigorosa análise microestrutural e ensaios mecânicos para, dessa forma, qualificá-las através de critérios usuais recomendados em normas que regulamentam a utilização deste material no reparo de estruturas. Foram investigadas as propriedades metalúrgicas através de microscopia ótica e eletrônica de varredura, fractografia e EDS (energy dispersive $x$-ray), além de ensaios de corrosão. Já as propriedades mecânicas foram averiguadas através de ensaios de impacto, de dobramento e perfis de microdureza. Com o intuito de garantir a qualidade da junta soldada e, por sua vez, a segurança da estrutura reparada, o processo FHPP atendeu aos requisitos recomendados pelas normas DNV-RP-FI I 2 e DNV-OS-FIOI. Palavras-chave: Reparo de estruturas; Aço inoxidável duplex; FHPP; Fases intermetálicas.

\section{FRICTION HYDRO PILLAR PROCESSING (FHPP) APLIED IN DUPLEX STAINLESS STEEL SAF 2205}

\begin{abstract}
The present investigation demonstrates the use of the Friction Hydro Pillar Processing (FHPP) to repair structures containing SAF 2205 (AID UNS S3 I803) duplex stainless steel (DSS) as the base material. Therefore, a preliminary study was carried out regarding the same material repaired with the Tungsten Inert Gas process (TIG). After obtaining the repaired joints in both processes, they underwent rigorous microstructural analysis and mechanical tests to qualify them through the usual criteria recommended in standards which regulate the use of this material, the DSS SAF 2205, in the repair of structures. The metallurgical properties were investigated by optical and scanning electronic microscopy, fractography and EDS (energy dispersive $\mathrm{x}$-ray) in addition to corrosion tests. The mechanical properties were investigated through impact tests, bending tests and microhardness profiles. With the purpose to guarantee the quality of the welded joint and, in turn, the safety of the repaired structure, the FHPP process met the requirements recommended by the standards DNV-RP-FII 2 and DNV-OS-FIOI. Keywords: Structural repair; Duplex stainless steel; FHPP; Intermetallic phases.
\end{abstract}

\section{INTRODUÇÃO}

O processo de soldagem por fricção com Pino consumível, que seria uma possível adaptação do nome registrado como "Friction Hydro Pillar Processing" (FHPP), é uma técnica relativamente recente de soldagem no estado sólido [I]. Com atrativo industrial para áreas do petróleo e gás, devido sua aplicação no reparo de descontinuidades do material, principalmente trincas, em estruturas offshore, tubulações de gás e petróleo, bem como na indústria

'Laboratório de Metalurgia Física - LAMEF, Departamento de Metalurgia - DEMET, Universidade Federal do Rio Grande do Sul - UFRGS, Porto Alegre, RS, Brasil.

${ }^{2}$ Instituto Federal de Educação, Ciência e Tecnologia do Rio Grande do Sul - IFRS, Caxias do Sul, RS, Brasil. E-mail: cleber.lessa@caxias.ifrs.edu.br

2176-1523 (c) 2017 Associação Brasileira de Metalurgia, Materiais e Mineração. Publicado pela ABM. Este é um artigo de acesso aberto distribuído sob os termos da licença Creative Commons CC BY-NC-ND (Attribution-NonCommercial-NoDerivs) - https:// creativecommons.org/licenses/by-nc-nd/4.0\%. 
naval [2]. Este processo consiste de duas etapas principais: uma primeira de furação e uma segunda de preenchimento. Nesta última, o consumível ou material de preenchimento, que é um Pino metálico equivalente ao material a ser reparado, é rotacionado em alta velocidade (geralmente acima de $1000 \mathrm{rpm}$ ) e posteriormente é prensado contra a chapa que pode ou não ter um furo de geometria similar ao Pino [3]. As etapas são demonstradas na Figura I.

$O$ atrito e a força axial fazem com que o Pino preencha o furo com grande penetração e consequente união metalúrgica [4]. Até então, os poucos estudos realizados para reparos por atrito via FHPP com baixa força não trataram de aços inoxidáveis duplex (AID), nesse será utilizado o SAF 2205. Este material tem tido um aumento considerável em sua utilização em plataformas, navios e equipamentos de indústrias químicas, de óleo e gás [5]. Os AID possuem uma estrutura mista de ferrita $(\alpha)$ e austenita $(\gamma)$, dessa

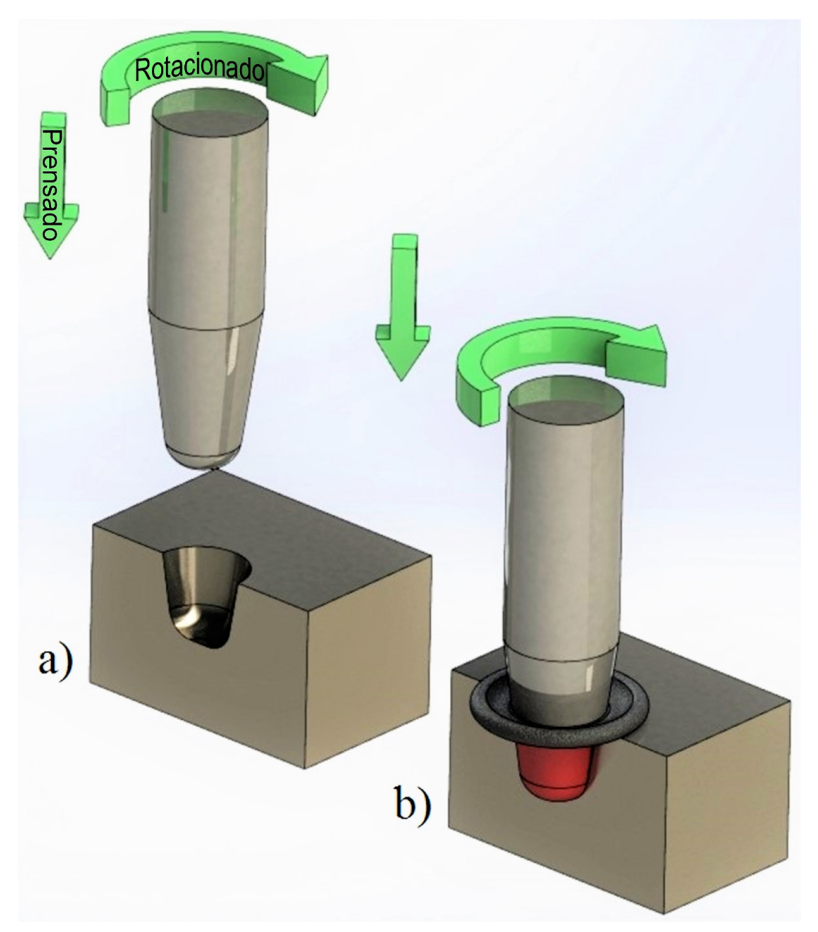

Figura I. Etapas e processamento. a) Um Pino consumível é rotacionado e prensado coaxialmente contra um furo pré-fabricado (usinado);

b) Devido a etapa anterior, ocorre mistura entre os materiais. forma, possuem uma combinação das propriedades dos aços inoxidáveis ferríticos e austeníticos [6].

Em certos períodos as estruturas industriais precisam ser restauradas devido aos desgastes e/ou falhas que ocorrem. O método mais efetivo utilizado para reparar estruturas duplex é a utilização de processo TIG, que geralmente apresenta melhores valores de ensaio de impacto comparado a outros processos de soldagem [7]. Porém, o processo TIG demanda utilização de gases, parada na planta de produção, além de poder vir a produzir fases frágeis e/ou carbonetos complexos, que geram riscos à integridade mecânica do material. Através do FHPP existem as vantagens de: poder reparar a estrutura com a planta e/ou o equipamento em funcionamento, pois não envolve fusão do material, logo possibilita uma baixa distorção estrutural além de ser um tipo de reparo tipicamente cirúrgico onde pouco material precisa ser removido para a execução do reparo. Além disso, o processo é considerado limpo por não gerar resíduos ambientais, devido à ausência gases nocivos e eliminação de respingos durante o processamento. Desta forma, as vantagens têm implicações econômicas, ambientais, metalúrgicas e mecânicas, pois através do FHPP pode-se alcançar grande integridade metalúrgica [8-I I].

O objetivo deste trabalho é investigar a utilização do FHPP, no AID SAF 2205, com baixa Força Axial de aplicação e o qualificar de forma semelhante aos reparos por processos convencionais de soldagem, através de ensaios padrões previstos em normas. Além disso, pretende-se ir além dos ensaios convencionais e trazer novas informações sobre fases precipitadas durante os processamentos, suas consequências na avaliação microestrutural e desempenho em corrosão.

\section{MATERIAIS E MÉTODOS}

A composição química do AID SAF 2205 e as propriedades mecânicas são apresentadas na Tabela I e na Tabela 2 respectivamente. Para a soldagem TIG, foi utilizada a vareta AWS SFA-5.9 ER 2209 (Tabela I).

$\mathrm{Na}$ Figura 2 são mostradas as medidas [mm] do Pino e da barra que servirá de metal base (MB). As geometrias e parâmetros para o processo FHPP foram escolhidos com base em estudos já realizados por diversos autores [1,2,4, I0, I2-14]. Importante destacar que a geometria do MB não é apropriada

Tabela I. Composição química da liga SAF 2205 e vareta consumível conforme fornecedor

\begin{tabular}{cccccccccccc}
\hline Material & $\mathbf{C}$ & $\mathbf{S i}$ & $\mathbf{M n}$ & $\mathbf{P}$ & $\mathbf{S}$ & $\mathbf{C r}$ & $\mathbf{M o}$ & $\mathbf{N i}$ & $\mathbf{C u}$ & $\mathbf{N b}$ & $\mathbf{N}$ \\
\hline SAF2205 & $\leq 0,03$ & $\leq 1,0$ & $\leq 2$ & $\leq 0,03$ & $\leq 0,02$ & $2 I-23$ & $2,5-3,5$ & $4,5-6,5$ & & 0,07 & $0,2-0,8$ \\
ER 2209 & $\leq 0,03$ & $\leq 0,9$ & $0,5-2,0$ & $\leq 0,03$ & $\leq 0,03$ & $21,5-23,5$ & $2,5-3,5$ & $7,5-9,5$ & $\leq 0,75$ & $0,2-0,8$ \\
\hline
\end{tabular}

Tabela 2. Propriedades mecânicas da liga SAF 2205

\begin{tabular}{cccc}
\hline Tensão de escoamento [MPa] & Tensão de ruptura [MPa] & Alongamento (\%) & Dureza Rockwell [HRC] \\
\hline 448 & 700 & 25 & 30 \\
\hline
\end{tabular}


para o processamento $T I G$, mas se optou por utilizar para manter as mesmas condições nos dois processos.

Após a obtenção do MB e do Pino consumível nas geometrias mencionadas, estes foram processados na máquina MSA 50 para soldagem FHPP. A MSA 50 é um equipamento desenvolvido no LAMEF (Laboratório de Metalurgia Física da Universidade Federal do Rio Grande do Sul) (Figura 3).

Parâmetros utilizados: Rotação, burn off (consumo do Pino), forjamento foram escolhidos após testes iniciais os seguintes valores: Força [kN]: 25; Velocidade de Rotação [rpm]: 7000; Força de Forjamento [kN]: 25; Tempo de Forjamento [s]: 10 Burn-off [mm]: 9. Para o processo TIG: Corrente [A]: 150; Tensão [V]: 220; Vazão Gás [1/min]: 17.

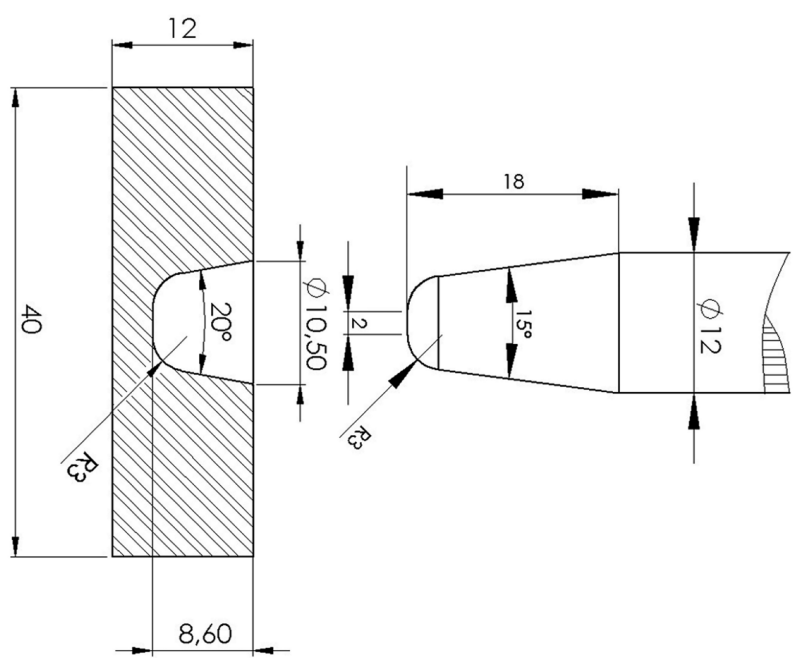

Figura 2. Croqui com as geometrias (unidade $\mathrm{mm}$ ) dos materiais confeccionados para os reparos.
Para revelar as fases, foi utilizado o reagente Behara modificado: $20 \mathrm{ml}$ de ácido clorídrico, $80 \mathrm{ml}$ de água destilada, $2 \mathrm{~g}$ de bifluoreto de amônio e I $\mathrm{g}$ de metabissulfito de potássio, por aproximadamente 5 segundos. Para visualização foi utilizado o microscópio ótico OLYMPUS modelo BX5 IM e microscópio eletrônico de varredura (MEV) SHIMADSU modelo SSX-550 Superscan, com sistema de análise de imagens e EDX acoplado.

Para avaliação quanto às características da união realizada por atrito se fez a avaliação dos percentuais de fases $\alpha$ e $\gamma$ (norma DNV-OS-FIOI [15]), avaliação do espaçamento interaustenítico (norma DNV-RP-FII2 [16]) e percentual de fases frágeis [17-19], todos os procedimentos conforme normas citadas. $O$ método utilizado para os percentuais de fases frágeis, foi através da análise pelo software Image ${ }^{\circledR}$.

Para o dobramento, foi utilizada a norma ASTME 190-08. Foi utilizada a máquina de ensaio eletromecânica Instron $8555 \mathrm{H}$ para o procedimento. $\mathrm{O}$ corpo de prova seguiu o modelo da ASTM A370. É sabido que as fases presentes no material influenciam na resistência ao impacto. $O$ ensaio de impacto Charpy foi realizado no equipamento INSTRON modelo SI- 103 instrumentado com capacidade de 406,7]. Foi necessária a macrografia das juntas soldadas para poder marcar a linha do entalhe do Corpo de Prova (CP). Como o processo FHPP não possui normas padrão para ensaios, - CP foi adaptado (Figura 4), conforme norma ASTM E8 (Figura 4). Esta adaptação teve como base estudos anteriores [20,21]. A norma referente ao ensaio foi a ASTM E23 [22].

A medição de microdureza foi através das zonas de soldagem entre $\circ$ MB e Pino para FHPP, MB e Zona de Fusão (ZF) para TIG. Estas medições foram feitas com o microdurômetro INSTRON Tukon 2100-B. Foi usada

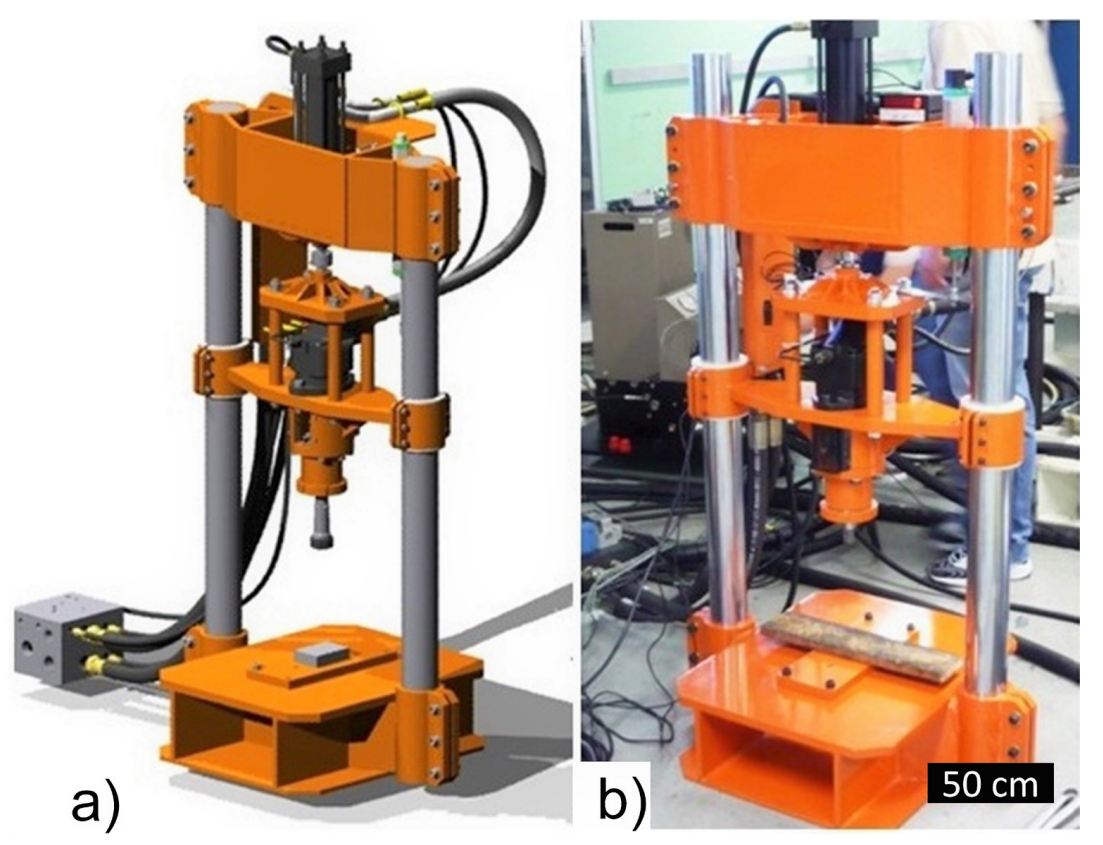

Figura 3. a) projeto da máquina; b) máquina pronta para processamento FHPP MSA 50. 


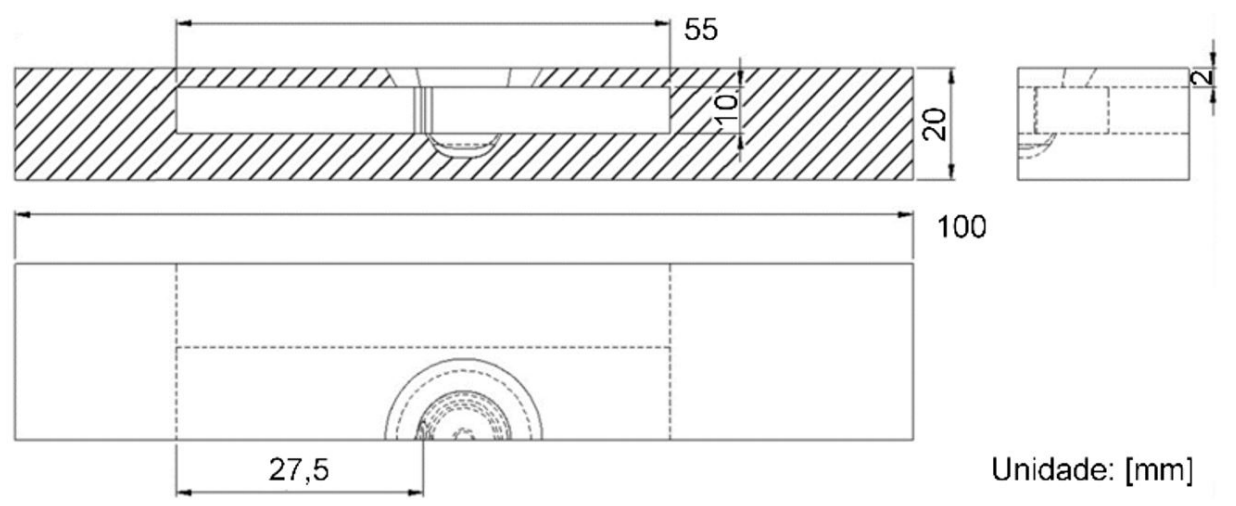

Figura 4. Desenho mostrando a extração do CP Charpy da junta soldada FHPP.

a escala HV0.5 com espaçamento de $0,25 \mathrm{~mm}$ entre as indentações por 10 segundos cada uma. Este ensaio levou em conta estudos anteriores [23,24]. A análise eletroquímica do material, avaliada através de curvas de polarização potenciodinâmica cíclica em solução $3,5 \% \mathrm{NaCl}$ (em peso) na temperatura ambiente, simulando meio marinho. Os CPs utilizados foram extraídos das amostras soldadas por FHPP e TIG, mais propriamente a Zona de Mistura-FHPP e a ZF-TIG. Ambos CPs foram usados como eletrodo de trabalho com área da superfície exposta de aproximadamente $0,5 \mathrm{~cm}^{2}$. O eletrodo de referência usado foi de calomelano saturado e um bastão de platina como contra-eletrodo.

\section{RESULTADOS}

A análise microestrutural dos materiais como recebido mostrou a presença das fases ferrita (fase escura) e austenita (fase clara) (Figura 5).

O acionamento da máquina começou com a velocidade rotacional. Após atingir a velocidade requerida, - Pino começou a ser prensado contra a base fixada até consumir a altura determinada no sistema (burn off). Como resultado, se obtém a produção de uma união pelo processo FHPP (Figura 6)

O tempo dos processamentos é resultado da sinergia dos parâmetros força, bun off e velocidade rotacional. Nota-se que quanto maior a força, menor o tempo (Figura 7) por acelerar o consumo do Pino. Após os processamentos, foram identificadas as zonas típicas do processo FHPP (Figura 8(a)) [25] para as análises que seguirão.

As zonas foram divididas em regiões: I, 2 e 3 (Figura 8(b)). Em seguida feitas micrografias referentes: região I: Figura 9(a), (b), (c); região 2: Figura 9 (d), (e), (f); região 3: Figura 9 (g), (h), (i). É perceptível a formação da austenita de Widmanstätten na ZM, Figura 9 entre (a) e (b) e (e) e (f).

Neste investigação, optou-se por fração do volume de $\alpha$ determinada por análise quantitativa de imagem por ser o método mais confiável [26]. (Tabela 3)
Nas zonas da junta soldada, ficou evidente que a quantidade da fase $\alpha$ está um pouco acima da quantidade encontrada inicialmente, tanto no MB quanto, no Pino, porém esta quantidade de fase e precisão relativa (\%RA) são aceitáveis conforme normas [15,16]. A análise mostrou uma razão $\alpha / \gamma$ balanceada e de acordo com as normas citadas. Segundo Lippold, taxas intermediárias de extração de calor $\left(\sim 50^{\circ} \mathrm{C} / \mathrm{s}\right)$ e um balanço entre $\alpha / \gamma$ resultam em uma boa tenacidade à fratura em materiais de base [2I]. Materiais com espaçamento pequeno entre fases tem maior resistência contra trincas induzidas por hidrogênio do que materiais com espaçamentos grandes. Um espaçamento $\gamma$ é considerado menos sensível a fragilização pelo hidrogênio quando menor que $30 \mu \mathrm{m}$ [16].

O parâmetro do ensaio de perfil utilizado para FHPP teve como base estudos anteriores $[1,23,27]$. O perfil de microdureza, representado pelas linhas pontilhadas na Figura 10 (a), mostra variação entre 250 e 290 HVO.5 na ZTMA-Pino.

Valores próximos da ZTA-Pino e ZTA-MB, ficaram entre 245 e $280 \mathrm{HV} 0.5$. É importante salientar que essas variações são consideradas baixas, pois o $\mathrm{MB}$ apresentou microdureza entre 240 e 250 HV0.5. É notável uma elevação da microdureza, quando se observa o perfil, na passagem da ZTMA-MB para ZM, provavelmente essa diferença ocorra devido a formação de austenita com formato de Widmanstätten na ZM e austenita secundária nucleada no interior da ferrita. Também ocorreu a diminuição da microdureza quando passa da ZTMA-Pino para a ZTA-Pino. Trabalhos anteriores $[8,10]$ mostraram que essa variação é comum nesse processo. $\mathrm{Na}$ Figura 10 (b) nota-se uma elevação da microdureza na passagem da ZTA-MB para ZF, que provavelmente ocorra devido a formação da $\gamma$-dendrítica (Figuras I I e |2). A seta na imagem (Figura I I) também mostra um pequeno defeito de soldagem, a falta de preenchimento.

Além disso, a macrografia obtida após a soldagem TIG (Figura I I) mostrou duas inclusões de tungstênio, com valor de dureza de $6 \mathrm{I} 2,5 \pm 56,8 \mathrm{HV}$, ocorrida provavelmente 

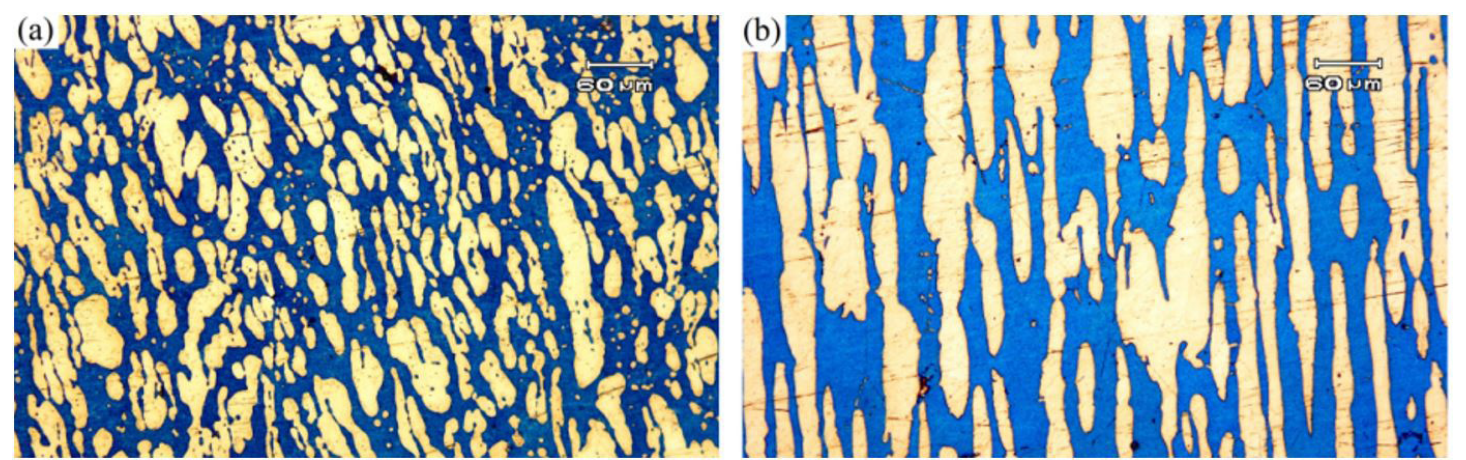

Figura 5. Material como recebido, ambos na direção de laminação. (a) barra chata para o MB; (b) barra redonda para os Pinos. Ataque: Behara modificado.
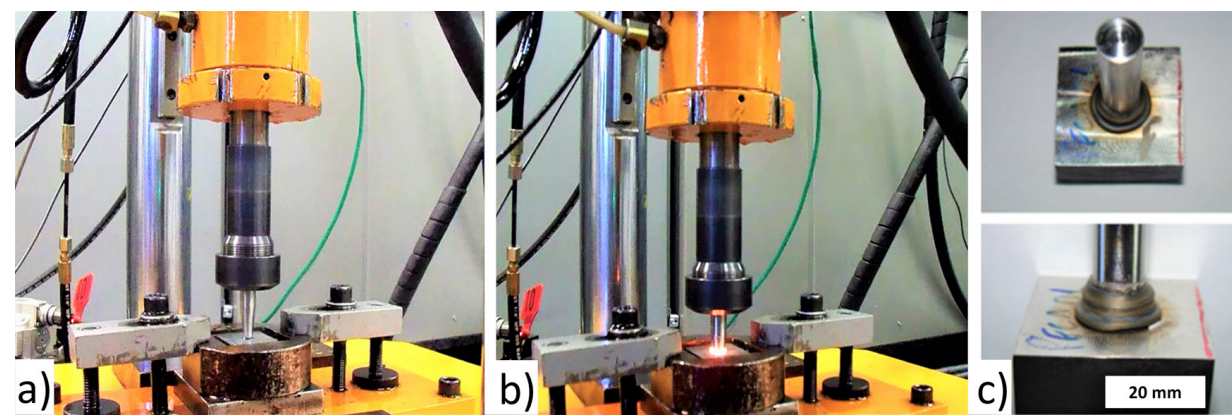

Figura 6. a) o pino sendo rotacionado; b) pino pressionado contra o $M B$; c) união.

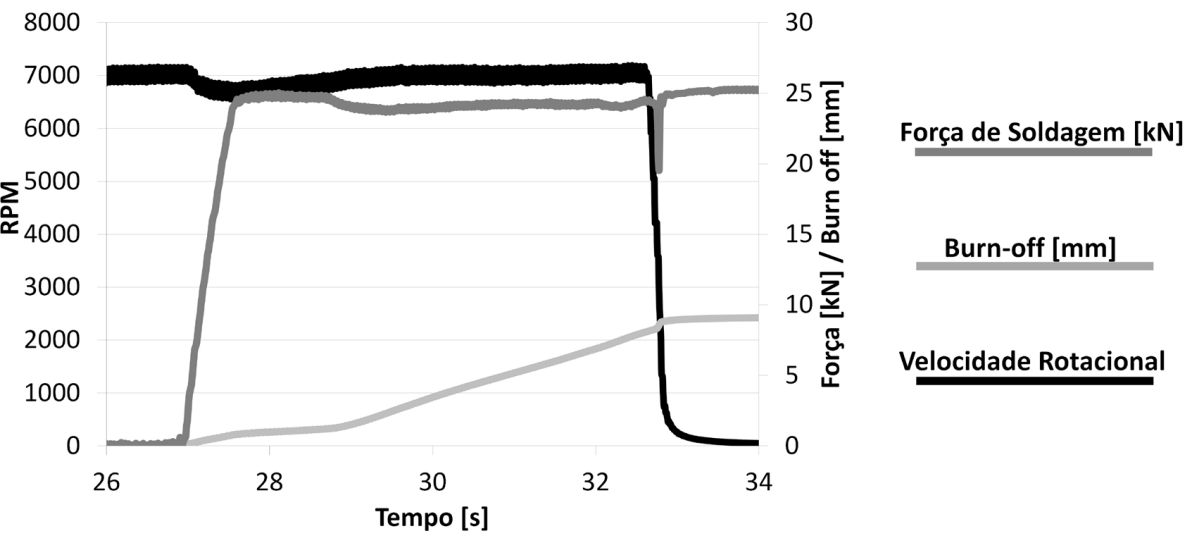

Figura 7. Aquisição dos dados conforme os parâmetros apresentados.

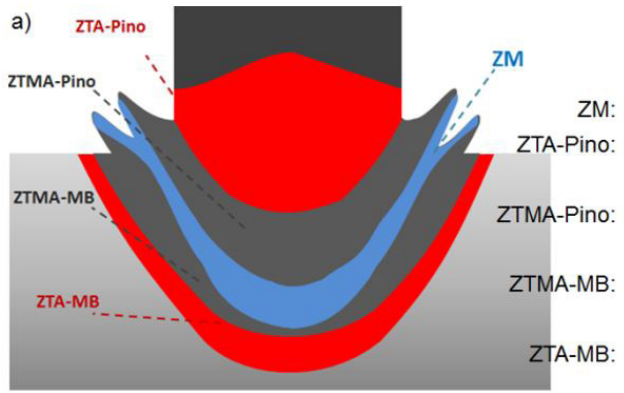

Zona de Mistura;

Zona Termicamente Afetada 6 no Pino;

Zona Termomecanicamente Afetada no Pino;

Zona Termomecanicamente

Afetada no Metal Base

Zona Termicamente Afetada

no Metal Base.

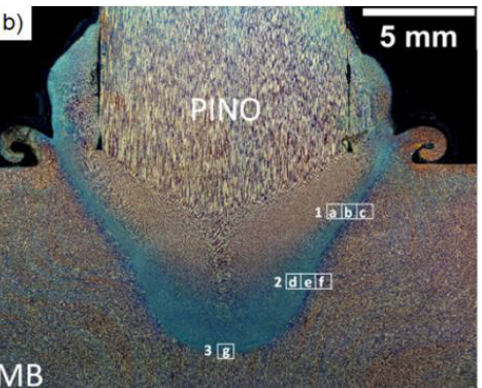

Figura 8. a) esquema mostrando as zonas formadas; b) macrografia. 

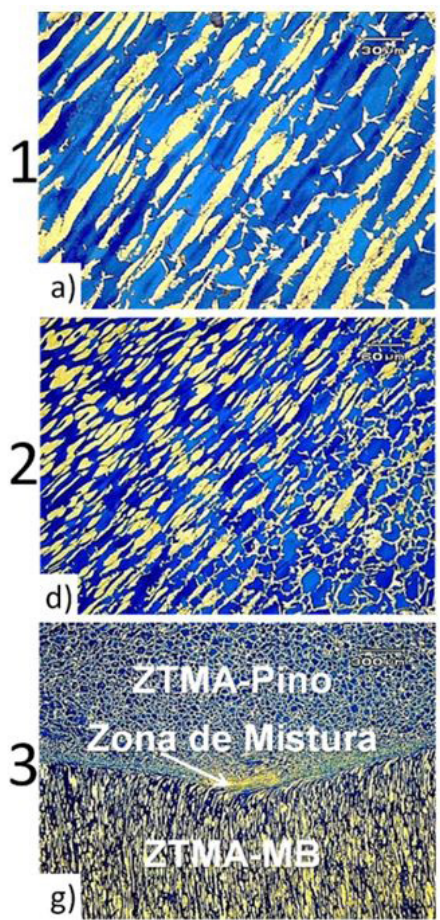
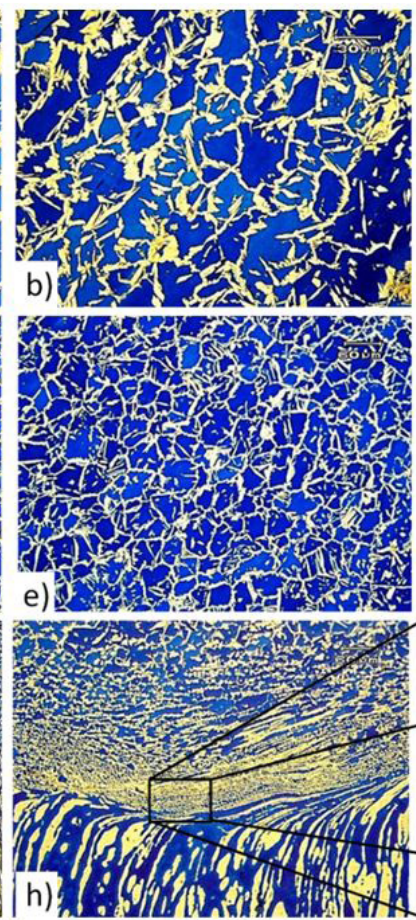
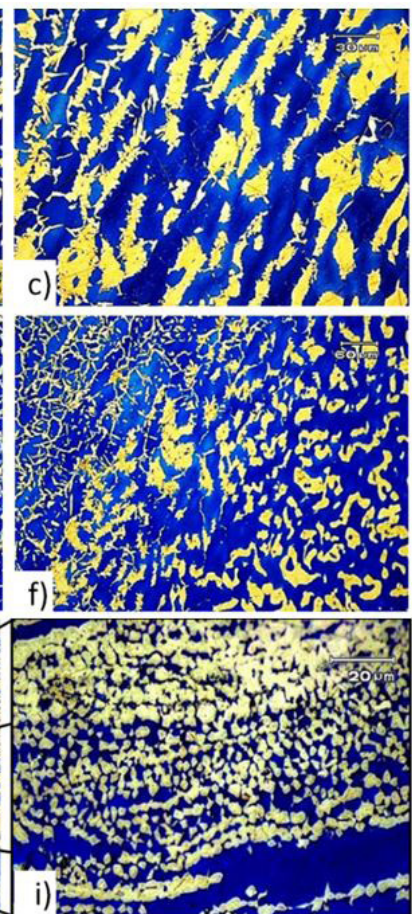

Figura 9. Micrografas referentes às regiões I, 2 e 3 da Figura 8 em diferentes profundidades. (a) e (d) são micrografias referentes a ZTMA-Pino, (b) e (e) micrografias referentes ZM, (c) e (f) micrografias referentes a ZTMA-MB. As micrografias (g), (h) e (i) mostram o detalhe da ligação na ponta do pino. Em todas as micrografias, fase $\alpha$ é a escura, fase $\lambda$ é a clara.

Tabela 3 - Percentuais de fases $\gamma$ e $\alpha$, espaçamento $\gamma$ para o processo FHPP

\begin{tabular}{|c|c|c|c|c|c|c|c|}
\hline Região & Zona & $\% \alpha$ & $\% \operatorname{RA} \alpha$ & $\% \gamma$ & $\% \mathbf{R A} \gamma$ & $\begin{array}{c}\text { Espaçamento } \\
\gamma(\mu \mathrm{m})\end{array}$ & $\begin{array}{c}\text { \%RA } \\
\text { Espaçamento } \\
\gamma\end{array}$ \\
\hline \multirow[t]{3}{*}{ I } & ZTMA-Pino & 57,63 & 9,32 & 42,37 & 7,94 & 10,39 & 4,47 \\
\hline & ZM & 67,69 & $|2,5|$ & 32,31 & 4,34 & 22,78 & 6,96 \\
\hline & ZTMA -MB & 61,17 & 5,75 & 38,83 & 5,96 & 14,76 & 3,34 \\
\hline \multirow[t]{3}{*}{2} & ZTMA-Pino & 64,70 & 7,25 & 35,3 & 5,12 & 18,83 & 5,82 \\
\hline & ZM & 61,09 & 11,54 & 38,91 & 6,07 & 19,26 & 7,38 \\
\hline & ZTMA -MB & 66,43 & 9,89 & 33,57 & 4,64 & 17,70 & 6,49 \\
\hline \multirow[t]{3}{*}{3} & ZTMA-Pino & 61,09 & 8,21 & 38,91 & 6,08 & 23,43 & 4,83 \\
\hline & ZM & 55,83 & 14,03 & 44,17 & 8,25 & 9,03 & 9,16 \\
\hline & ZTMA -MB & 59,98 & 7,18 & 40,02 & 7,76 & 22,43 & 8,65 \\
\hline \multirow{3}{*}{$\begin{array}{l}\text { Material não } \\
\text { processado }\end{array}$} & Pino Longitudinal & 49,63 & 4,80 & 50,37 & 4,93 & 31,54 & 6,73 \\
\hline & Pino Transversal & 55,01 & 5,28 & 44,99 & 4,46 & 24,43 & 6,80 \\
\hline & $\mathrm{MB}$ & 49,69 & 7,56 & 50,31 & 7,39 & 25,76 & 5,14 \\
\hline
\end{tabular}
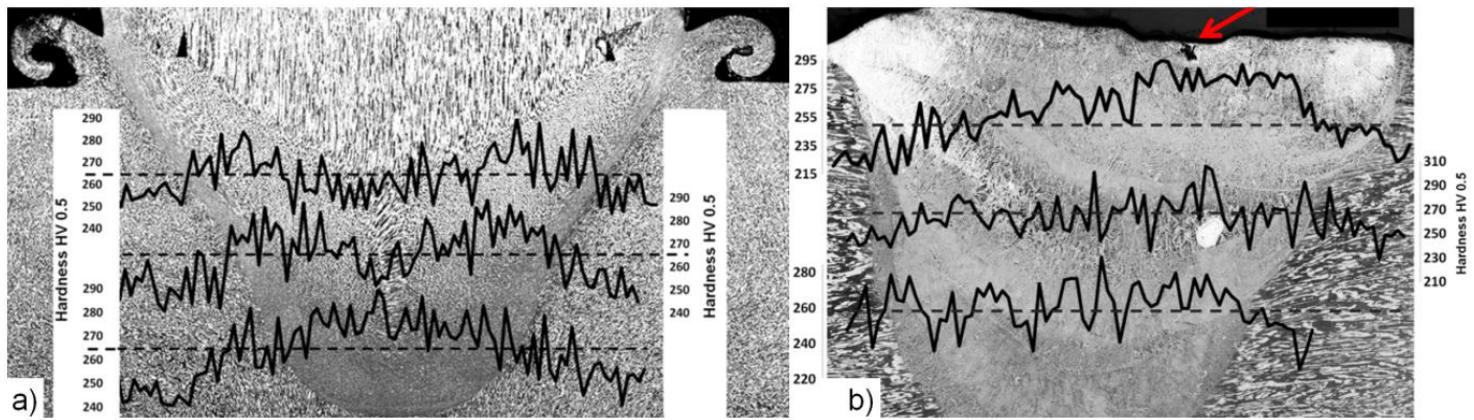

Figura 10. Perfil de microdureza [HV]. a) FHPP; b) TIG. 

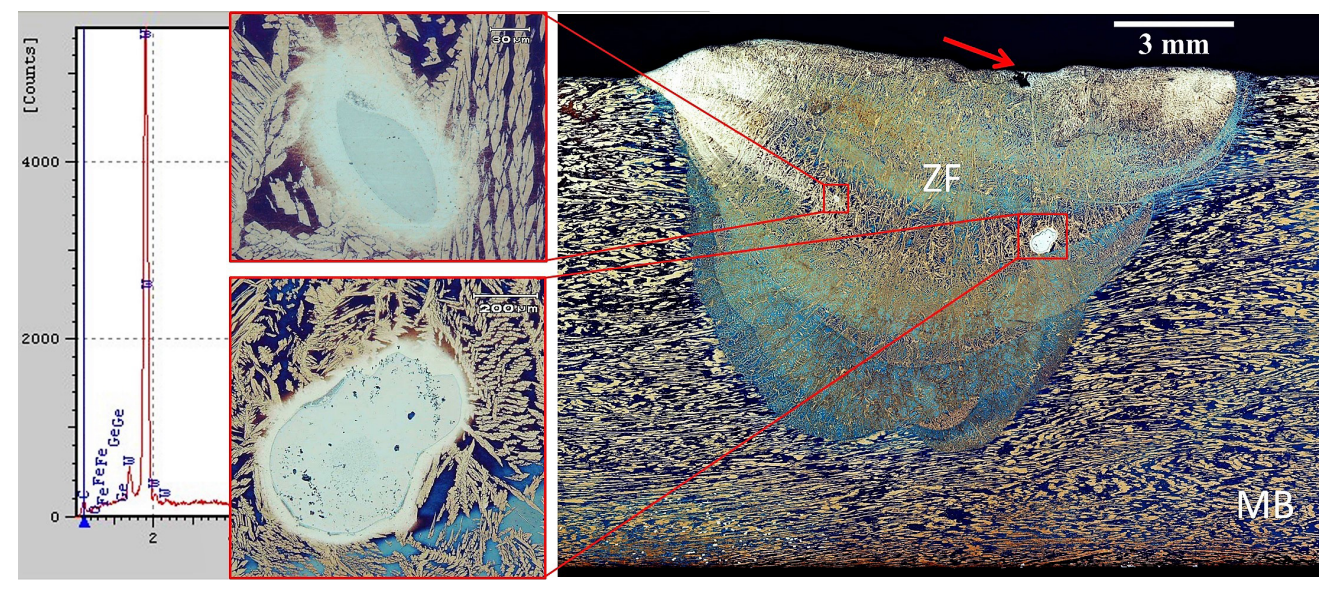

Figura I I. Macrografia. ZF: zona fundida; MB: Metal de Base. Detalhe: inclusão de W.

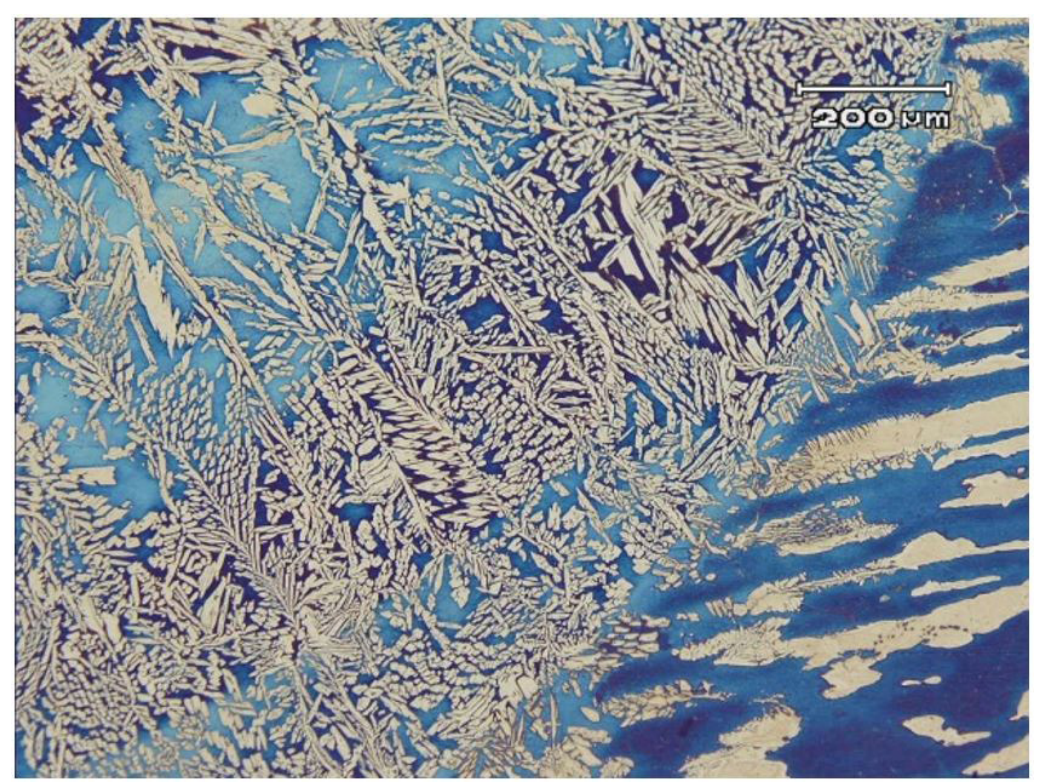

Figura 12. Micrografia. Detalhe: interface do MB e ZF. Microestrutura predominante de $\gamma$-dendrítica.

com a quebra do eletrodo devido à alta corrente necessária para executar a soldagem.

Conforme norma para o ensaio de impacto [28], para o material em questão, o valor mínimo é de 54J de energia de impacto. Para este estudo foram realizados quatro ensaios para cada condição estudada. Os resultados obtidos foram similares: $234,00 \mathrm{~J} \pm 29$ (TIG); 237J \pm 37 (FHPP). Com relação ao dobramento, no CP FHPP, não houve qualquer tipo de descontinuidade. Com os parâmetros estudados, para o CP TIG, após o dobramento houve formação de descontinuidades e, dessa forma, a norma permite afirmar que a solda não produziu união de qualidade (Figura I3).

No ensaio de corrosão, ficou evidente que a microestrutura formada na Zona de Mistura (ZM-FHPP) permitiu um melhor comportamento que no Metal de
Solda (MS-TIG). Fato esse constatado pela observação no gráfico da Figura I4, onde se observa uma maior densidade de corrente do MS-TIG em relação ao ZM-FHPP mesmo quando se varia o potencial de aproximadamente $-500 \mathrm{mV}$ até aproximadamente $1300 \mathrm{mV}$.

Foi constatada a presença da fase $\chi$ (Figura I5) identificada por Elétrons retroespalhados no MEV (Figura I5 (d)) por contraste devido ao fato da fase $\chi$ ser rica em Mo $[26,29]$. Conforme procedimento [15] numa magnificação de 400x é aceitável um percentual máximo de $0,5 \%$ fases frágeis precipitadas [17-19]. No MB e na zona de mistura do processo FHPP estas fases não foram encontradas. A análise criteriosa detectou a região "I" (Figura 8(b)) e a ponta do Pino como sendo as mais propícias para tal formação, mais precisamente entre a ZTA-Pino e a ZTMA-Pino. Para TIG, 


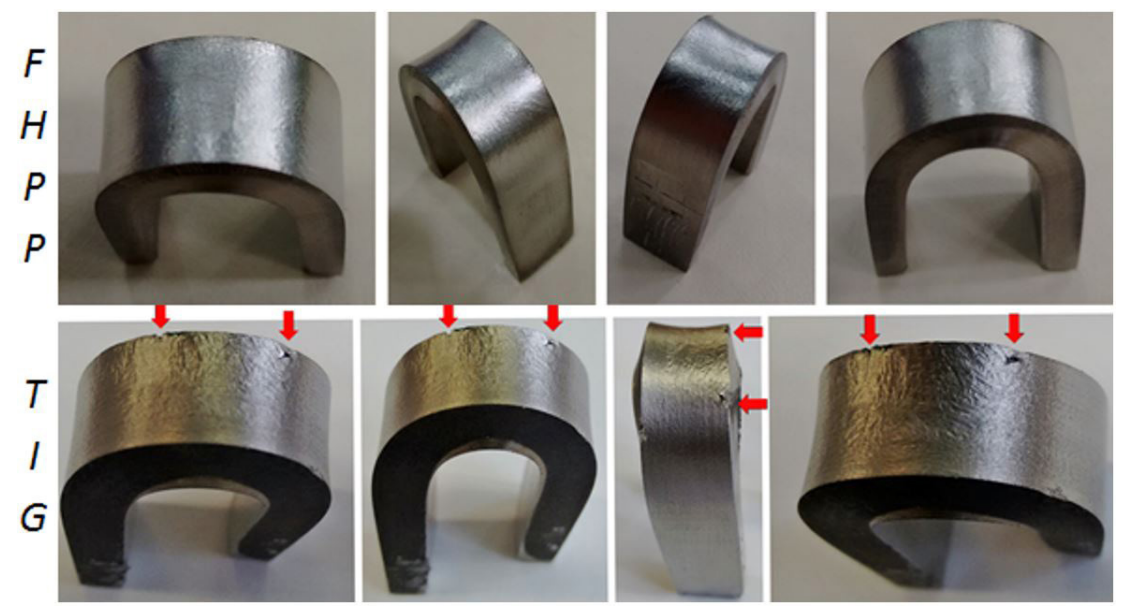

Figura I3. CP de dobramento sob diferentes ângulos de visão. As setas mostram as falhas.

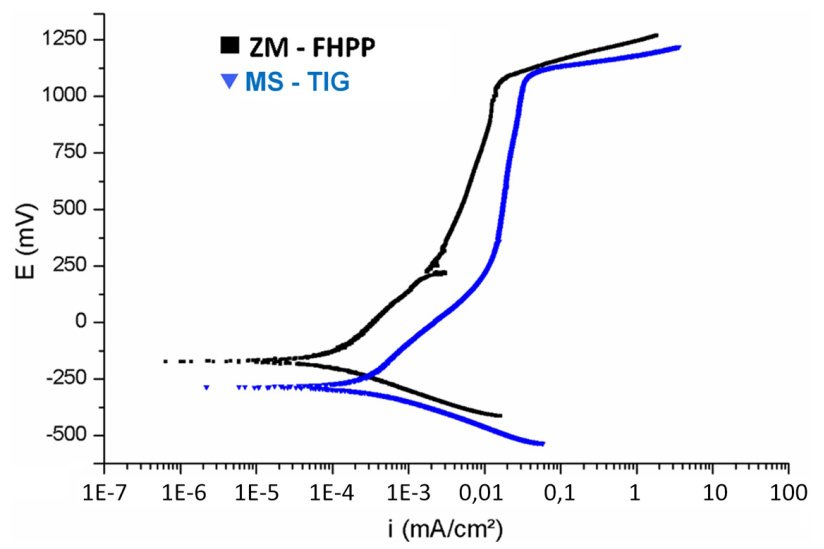

Figura 14. Curva de polarização cíclica dos CPs ZM-FHPP e ZF-TIG.
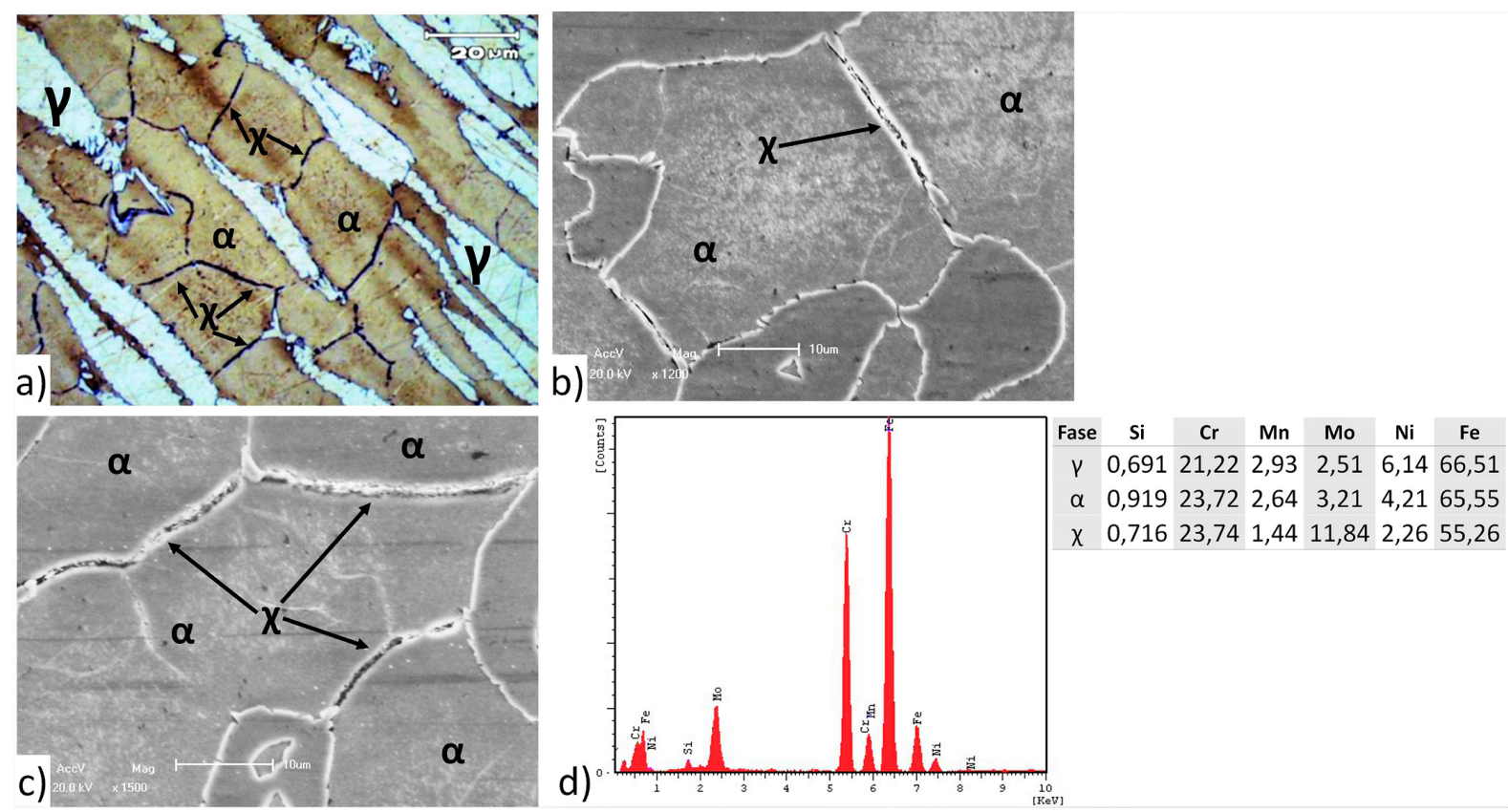

Figura I5. Micrografias com destaque na fase $\chi$ ZTMA-MB (FHPP). (a) Microscópio ótico, (b) e (c) MEV. (d) EDS das fases $\gamma, \alpha$ e $\chi$ (\% em peso). Ataque: Behara modificado. 

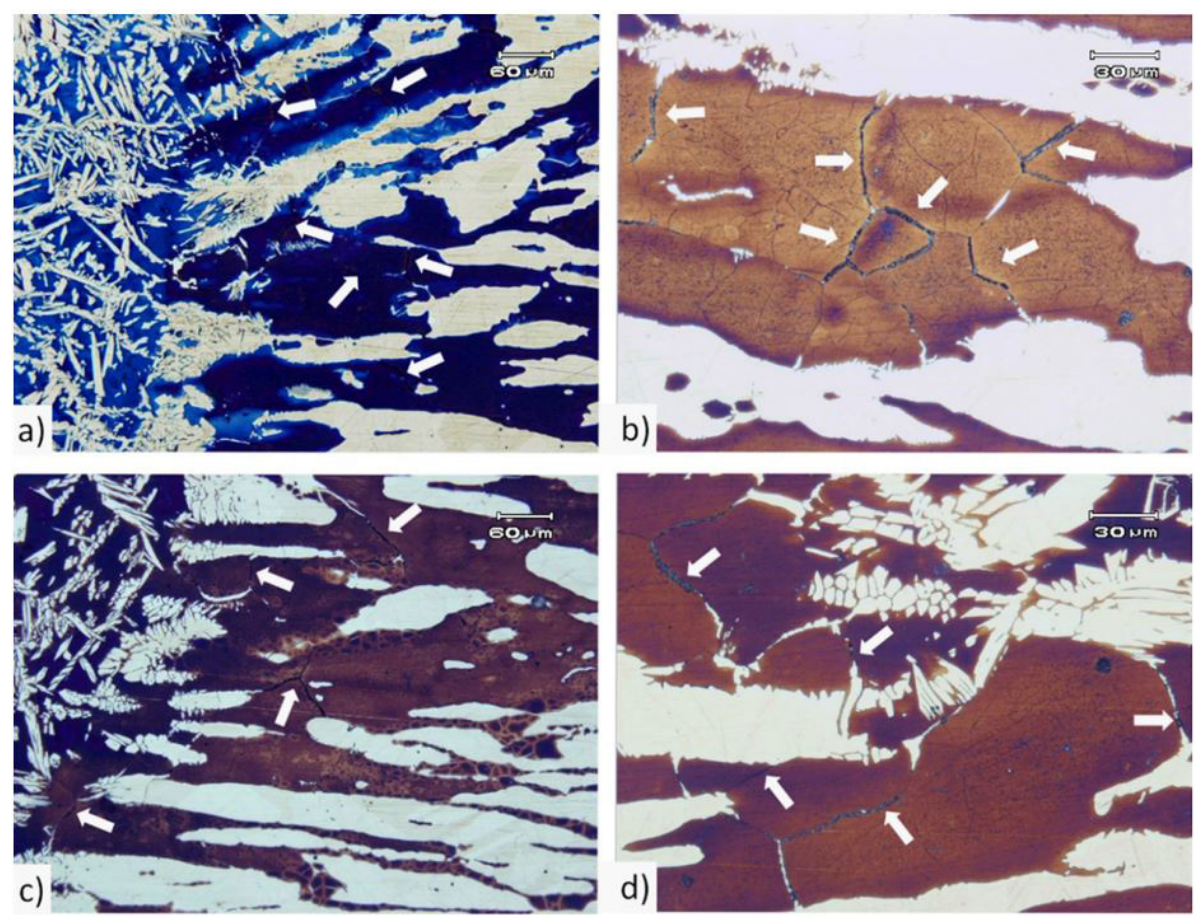

Figura 16. Micrografa. As setas brancas mostram fases precipitadas em diferentes regiões do processo TIG. (a) ZTMA-MB contendo a fase precipitada $\chi$ no contorno do grão da fase $\alpha$ (escura). (b) $\chi$ em maior aumento na ZTMA-MB. (c) Outra amostra da ZTMA-MB que também mostra $\chi$. (d) $\chi$ no contorno de $\alpha$ (escura). Ataque: Behara modifcado.

foram encontradas fases frágeis em quantidade acima do aceitável em toda a interface entre a ZF e o MB (Figura I6), provavelmente devido ao elevado aporte térmico utilizado durante a soldagem.

\section{DISCUSSÃO}

Precipitados fragilizantes foram encontrados em maiores quantidades nas amostras soldadas pelo processo TIG. Foi evidenciado também que estes se encontram em todas as regiões da junta soldada, enquanto que no processamento FHPP apenas na região "I" e na ponta do Pino sendo estas as mais propícias. Além disso, quando se comparou regiões semelhantes dos dois processos, percebeu-se que no processo TIG ocorre a formação de precipitados maiores e, por consequência, uma maior possibilidade de formação de fase $\sigma$ [26].

A cinética de precipitação da fase $\sigma$ é acelerada se atingida à temperatura necessária e é a fase que mais prejudica, pois interfere negativamente na resistência à corrosão e na tenacidade pelo aumento da dureza [30,3I]. Estas reações de precipitações modificam o balanço original PRE entre as fases, e a ferrita transformada se torna mais propícia para a corrosão localizada. A principal deterioração da estabilidade do filme passivo e a resistência ao pite são ambos causados pela diminuição do $\mathrm{Cr}$ e Mo durante a formação de $\sigma$ [32]. A precipitação destas fases afeta, tanto as propriedades mecânicas, quanto as propriedades de corrosão, principalmente a localizada [33].

\section{CONCLUSÕES}

I. Foi possível obter uma junta soldada livre de defeitos com o AID SAF 2205 para os parâmetros da força aplicada de $25 \mathrm{kN}$, da velocidade de $7000 \mathrm{RPM}$, do burn-off $9 \mathrm{~mm}$ e do forjamento pelo período de 10 segundos, com geometria contendo curvatura com raio $3 \mathrm{~mm}$ tanto na ponta do Pino quanto no canto do furo no $M B$, altura do furo no $M B 8,6 \mathrm{~mm}$ e do cone do Pino de $18 \mathrm{~mm}$;

2. Os percentuais de fases frágeis após processamento FHPP estavam dentro dos limites aceitáveis pelas normas DNV-RP-FII 2 e DNV-OS-FI0I. Porém, para o processo $T I G$, estas fases frágeis não estavam dentro dos limites aceitáveis possivelmente pelo elevado aporte térmico utilizado na soldagem;

3. As descontinuidades averiguadas, inclusão de tungstênio, falta de fusão e falta de preenchimento e falha durante o dobramento podem ser devido ao operador, parâmetros e geometria da junta utilizada. Seria necessário testar outros parâmetros para o processo TIG. O processamento FHPP pode ser automatizado, enquanto que para o processo TIG com fins de reparo de estruturas, não é possível, logo, a qualidade dependerá do profissional atuante;

4. Houve uma variação maior no perfil de microdureza no processo TIG, em comparação com o processamento FHPP. A microestrutura formada devido ao processo 
FHPP variou de grãos equiaxiais alongados com uma razão $\alpha / \gamma$ balanceada, para $\gamma$-Widmanstätten, enquanto que no processo TIG ocorreu a formação microestrutural $\gamma$-dendrítica. $O$ espaçamento $\gamma$ foi considerado pequeno quando se processa por FHPP, e conforme normas esse fato garante grande resistência contra trincas induzidas por hidrogênio. Porém, para o processo TIG não foi possível medir devido a microestrutura indesejável $\gamma$-dendrítica;

5. Portanto, diante de todas as averiguações sobre o material e parâmetros deste estudo, houve a comprovação da possibilidade de utilização do FHPP como mais um processo com potencial para ser utilizado na manutenção de estruturas de petróleo e gás, papel e celulose, e demais indústrias químicas.

\section{Agradecimentos}

Os autores querem agradecer ao professor Telmo Roberto Strohaecker (in memoriam) ao apoio dado pelo LAMEF e colaboradores, em especial Diogo Busati, Francisco Bandeira, Facundo Lopez e Rafael Eugênio.

\section{REFERÊNCIAS}

I Meyer A. Friction hydro pillar processing - bonding mechanism and properties. Springer: Wilhelm-Johnen-Strasse; 2003. GKSS School of Environmental Research Series.

2 Jardim MP, Gonçalves RA, Paes MTP, Pires RR, Franco VLDS. Efeito da geometria do pino e do furo no reparo por atrito resultados preliminares. Tecnol Em Metal E Mater. 2007;4(2):27-32.

3 Lessa CR L, Martinazzi D, Figueiredo AP, Machado RB, Fanezi C, Strohaecker T. Microstructural behavior of SAF 2205 Duplex Stainless Steel Welded by Friction Hydro Pillar Processing. Materials Research. 2016;19(4):928-936.

4 Yeh FWT, Pereira da Cunha PHC, Lessa CR L, Clarke T, Strohaecker T. Evaluation of Discontinuities in A36 Steel Repairs with Friction Hydro Pillar Processing Using Different Axial Forces. ISIJ International. 20I3;53(I2):2269$227 \mathrm{I}$.

5 Alvarez-Armas I, Degallaix-Moreuil S. Duplex stainless steels. Hoboken: John Wiley \& Sons Inc.; 2009.

6 Colpaert H. Metalografia dos produtos siderúrgicos comuns. 4. ed. Brasil: Blucher; 2008. 672 p.

7 Folkhard E, Rabensteiner G, Perteneder E, Schabereiter H, Tösch J. Welding metallurgy of stainless steels. New York: Springer-Verlag Wien; 1988.

8 Ambroziak A, Gul B. Investigations of underwater FHPP for welding steel overlap joints. Archives of Civil and Mechanical Engineering. 2007;7(2):67-76.

9 Blakemore G. Friction welding - technology for the new millennium. In: Proceedings of The Offshore Technology Conference; 1999; Houston, Texas. Richardson: One Petro; 1999 [acesso em 2013 Abr I2]. Disponível em: http:// www.onepetro.org/mslib/servlet/onepetropreview?id=OTC-I I063-MS

10 Meyer A, Pauly D, Santos JF, Pinheiro G, Roos A, Gibson D, et al. Considerations on robotic friction stitch welding for the repair of marine structures. In: Proceedings of The 20th International Conference on Offshore Mechanics and Arctic Engineering - OMAE; 200 I June 3-8; Rio de Janeiro. New York: American Society of Mechanical Engineers; 2001. p. I45-I5I.

I I Santos GM, Formoso CM, Franco SD, Franco VLDS. Optimization of control parameters in a friction hydro pillar processing unit to repair oil steel structures. Journal of the Brazilian Society of Mechanical Sciences and Engineering. 2012 [acesso em 26 set. 20I4];5:572-582. Disponível em: http://www.abcm.org.br/pt/wp-content/anais/ cobem/20I I/PDF/265802.PDF

12 Baosheng Z, Xiangdong J, Jiaqing C, Zong YQ. Numerical simulation onto the preliminary period of Friction Hydro Pillar Processing in Friction Stitch Welding. In: Proceedings of the International Conference on Mechanic Automation and Control Engineering - MACE; 2010 Jun 26-28; Wuhan, China. Piscataway: IEEE Conference Publications; 20 I0. p. 5617.

13 Pires RR. Efeitos da geometria, da força axial e da rotação no reparo por atrito. Uberlândia: Faculdade de Engenharia Mecânica, Universidade Federal de Uberlândia; 2007.

I4 Santos GM, Formoso CM, Franco SD, Franco VLDS. Optimization of control parameters in a friction hydro pillar processing unit to repair oil steel structures. Rio de Janeiro: ABCM; 2012. (ABCM Symposium Series in Mechatronics; vol. 5). 
15 Det Norske Veritas. Submarine pipeline systems: DNV-OS-FI0I. Oslo: DNV; 2012.

16 Det Norske Veritas. Design of duplex stainless steel subsea equipment exposed to cathodic protection: DNV-RP-FI I2. Oslo: DNV; 2008.

17 E04 Committee. Practice for determining the inclusion or second-phase constituent content of metals by automatic image analysis [internet]. West Conshohocken: ASTM International; 2008 [acesso em I5 out. 20 I4]. Disponível em: http://www.astm.org/doiLink.cgi?EI245

18 E04 Committee. Test method for determining volume fraction by systematic manual point count [internet]. West Conshohocken: ASTM International; 201 I [acesso em I5 out. 20I4]. Disponível em: http://www.astm.org/doiLink. cgi?E562

19 E04 Committee. Test methods for determining average grain size [internet]. ASTM International; 2013 [acesso em 4 out. 2014]. Disponível em: http://www.astm.org/doiLink.cgi?EII 2

20 Cui L, Yang X, Wang D, Hou X, Cao J, Xu W. Friction taper plug welding for S355 steel in underwater wet conditions: Welding performance, microstructures and mechanical properties. Materials Science and Engineering A. 2014;611:15-28.

2I Lippold JC, Varol I. Baeslack W III. Composition and microstructure on the haz toughness of duplex stainless steels at -20C. Welding Journal. 1994:75-80

22 American Society for Testing and Materials. Notched bar impact testing of metallic materials: ASTM E23 - I2C. West Conshohocken: ASTM; 2012.

23 Cui L, Yang X, Wang D, Cao J, Xu W. Experimental study of friction taper plug welding for low alloy structure steel: Welding process, defects, microstructures and mechanical properties. Materials \& Design. 2014;62:27I-28I.

24 Unfried SJ, Paes MTP, Hermenegildo TFC, Bastian FL, Ramirez AJ. Study of microstructural evolution of friction taper plug welded joints of C-Mn steels. Science and Technology of Welding and Joining. 2010;15(6):506-5 I 3.

25 Unfried J, Hermenegildo TFC, Paes MTP, Pope A, Ramirez AJ. Influence of Process Parameters in the TMAZ Microstructural Evolution of C-Mn Steels Friction Hydro-Pillar Welded Joints. In: Proceedings of The 8th International Conference of Trends Welding Research; I-6 June 2008; Georgia. Ohio: ASM; 2008; p. 38I-384.

26 Escriba DM, Materna-Morris E, Plaut RL, Padilha AF. Chi-phase precipitation in a duplex stainless steel. Materials Characterization. 2009;60(II):1214-1219.

27 Lessa CR L. Soldagem FHPP - processo e metalurgia nas transformações das fases de um aço C-Mn. Porto Alegre: UFRGS; 2011.

28 American Society for Testing and Materials. Standard test methods for detecting detrimental intermetallic phase in duplex austenitic/ferritic stainless steels: ASTM A923-I4. West Conshohocken: ASTM; 2014.

29 Chen T, Weng K, Yang J. The effect of high-temperature exposure on the microstructural stability and toughness property in a 2205 duplex stainless steel. Materials Science and Engineering A. 2002;338(I-2):259-270.

30 Gunn R. Duplex stainless steels: microstructure, properties and applications. Illinois: Woodhead Publishing Elsevier; 2003. $216 \mathrm{p}$.

3I Totten G. Steel heat treatment: metallurgy and technologies. Boca Raton: Taylor \& Francis Group; 2006. 832 p.

32 Angelini E, De Benedetti B, Rosalbino F. Microstructural evolution and localized corrosion resistance of an aged superduplex stainless steel. Corrosion Science. 2004;46(6): I35 I- I 367.

33 Pohl M, Storz O, Glogowski T. Effect of intermetallic precipitations on the properties of duplex stainless steel. Materials Characterization. 2007;58(I):65-7I.

Recebido em: 3 Jun. 2017

Aceito em: 6 Nov. 2017 